\title{
Mucormycosis in a Tertiary Care Center in South India: A 4-Year Experience
}

\author{
Poorna Priya ${ }^{1}$, Vithiya Ganesan ${ }^{2}$, T Rajendran $^{3}$, VG Geni ${ }^{4}$
}

\begin{abstract}
Background: Mucormycosis is an emerging fungal infection in both developed and developing countries with different target population. Unusual isolates and unusual clinical presentations have been reported from India in recent times.

Objectives: The present study was done to know the epidemiology, risk factors, diagnostic modalities, and treatment outcome of mucormycosis patients in our hospital.

Materials and methods: The study was carried out over a period of 4 years from October 2015 to October 2019. This is a retrospective observational chart review of patients diagnosed with proven and probable mucormycosis. Information on demography, clinical features, risk factors, laboratory and radiological findings, treatment (including medical and surgical treatment), and outcome was extracted from the records. Primary outcome at 42 days was determined.

Results: Thirty-eight patients were diagnosed with mucormycosis based on microbiological and/or histopathological examination (HPE) of the clinical samples. Most commonly affected age-group was between 41 years and 60 years. More number of cases were reported during the months of post rainy season (September to December). A large number of patients (77\%) presented with uncontrolled diabetes mellitus. Rhino-orbito-cerebral mucormycosis was the most common presentation followed by cutaneous infection. Antifungal treatment was started in around $80 \%$ of the cases. Twenty patients were managed by combined medical and surgical intervention. Eight patients died while the outcome of nine was unknown, as they left the hospital against medical advice and could not be followed up.

Conclusion: Mucormycosis, an insidious killer, should be an important differential diagnosis in progressive soft tissue infections and deep organ infection.

Keywords: Amphotericin B, Mucorales, Surgical debridement.

Indian Journal of Critical Care Medicine (2020): 10.5005/jp-journals-10071-23387
\end{abstract}

\section{INTRODUCTION}

Mucormycosis is an emerging serious healthcare problem in developed and developing countries. New species are emerging and uncommon isolates and unusual clinical presentations such as isolated renal mucormycosis are being reported from India in recent times. Many studies have been reported from disparate regions of India but have been largely restricted to a few large medical centers, presumably where identification is possible. There is paucity of data from South Tamil Nadu. So the present study was done to know the epidemiology, risk factors, diagnostic modalities, and the treatment outcome of mucormycosis patients in our hospital.

\section{Materials and Methods}

The present study was conducted in the Department of Microbiology, Velammal Medical College Hospital, Microbiology, in association with the Department of Pathology and other clinical departments from which samples were received to find the prevalence of mucormycosis in our region. The study was carried out over a period of 4 years from October 2015 to October 2019. This is a retrospective observational chart review of patients diagnosed with proven and probable mucormycosis according to revised European Organization for Research and Treatment of Cancer/ Infectious Diseases Mycoses Study Group Consensus Group criteria. Diagnosis of definite mucormycosis was made on demonstration of aseptate ribbon-like broad hyphae on histopathology of biopsied sample with or without culture confirmation. Probable mucormycosis was diagnosed when patient had suggestive clinical criteria including clinical symptoms with radiological confirmation
${ }^{1}$ Department of Pathology, Velammal Medical College Hospital and Research Institute, Madurai, Tamil Nadu, India

${ }^{2-4}$ Department of Microbiology, Velammal Medical College Hospital and Research Institute, Madurai, Tamil Nadu, India

Corresponding Author: Vithiya Ganesan, Department of Microbiology, Velammal Medical College Hospital and Research Institute, Madurai, Tamil Nadu, India, Phone: +91 9486312483, e-mail: vidhya.md@gmail.com

How to cite this article: Priya P, Ganesan V, Rajendran T, Geni VG. Mucormycosis in a Tertiary Care Center in South India: A 4-Year Experience. Indian J Crit Care Med 2020;24(3):168-171.

Source of support: Nil

Conflict of interest: None

of lesion [on computed tomography (CT) scan/magnetic resonance imaging] and Mucorales were isolated from nonsterile samples (e.g., bronchoalveolar lavage, biopsied/curette sample from nasal area). Information on demography, clinical features, risk factors, laboratory and radiological findings, treatment (including medical and surgical treatment), and outcome was extracted from the records. Primary outcome at 42 days was determined. Ethics committee approval was taken for this study, and written informed consent was obtained from patients whose clinical images are used.

\section{Results}

Over a study period of 4 years, a total of 38 patients were diagnosed with mucormycosis based on microbiological and/or HPE of the 
clinical samples. Demographic and clinical features of the patients are presented in Table 1. Of the total patients, 27 were males and 11 were females. Most of them were adults, except 1 who was 16 years old. The most commonly affected age-group was that between 41 and 60 years. A large number of patients $(n=29)$ presented with diabetes mellitus. Two were newly diagnosed and three patients presented with diabetic ketoacidosis.

Rhino-orbito-cerebral mucormycosis $(n=28)$ was the most common presentation followed by cutaneous infection $(n=8)$, one renal, and one pulmonary mucormycosis. In four patients, culture was not done; and in 25 of 34 cases, fungus grew on culture. While in the others, the diagnosis was made either on direct potassium hydroxide (KOH) wet mount (all 38 cases) or on the HPE (19 of 22 ROC cases, $3 / 3$ cutaneous infection, renal, and pulmonary were positive by histopathology). There were 30 cases of definite and 8 cases of probable mucormycosis. Most common fungus was Rhizopus oryzae followed by Apophysomyces variabilis, Mucor spp., Lichtheimia corymbifera, and two unidentified isolates. Failure to sporulate was the major hindrance in the identification of isolates. Distribution of various mucormycetes with various clinical presentations is given in Table 2.

Antifungal treatment was initiated in 30 patients, 29 of them were managed with amphotericin B (either conventional or liposomal formulation). One patient was managed with a combination of liposomal amphotericin B and oral posaconazole.

Table 1: Demographic and clinical features of patients

\begin{tabular}{lll}
\hline $\begin{array}{l}\text { Parameters } \\
\text { Age in years, median } \\
\text { (range) }\end{array}$ & $n=38$ \\
Sex & Male & $46(16-75)$ \\
& Female & 28 \\
Risk factors & DM & 10 \\
& Trauma & 22 \\
& Hematological malignancy & 2 \\
& Hypertension & 4 \\
Site involved & Chronic kidney disease & 2 \\
& ROC & 28 \\
& Cutaneous & 8 \\
& Pulmonary & 1 \\
Antifungal treatment & Isolated renal & 1 \\
& ABDC & 5 \\
& L AmB & 25 \\
Surgery in ROC & Posaconazole & 1 \\
& Radical resection & None \\
& Sinoscopic debridement & 11 \\
\hline
\end{tabular}

DM, diabetes mellitus; $A B D C$, amphotericin $B$ deoxycholate; $L A m B$, liposomal amphotericin B
Among medically managed patients, liposomal amphotericin B was given for 25 patients. Conventional amphotericin $B$ was given only for five patients. Patients who recovered received an additional 2 weeks to 4 weeks of amphotericin B depending on the clinical condition. Treatment and outcome details are given in Table 3. Despite treatment, eight patients died of complications such as cardiorespiratory collapse and septic shock. In nine patients, no treatment was given, as some patients were not able to afford the treatment and left against medical advice, despite explaining the dire consequences. A total of 20 patients were managed by combined medical and surgical intervention, of which 11/28 ROC patients were managed by functional endoscopic sinus surgery, all cutaneous cases $(n=8)$ underwent repeated debridement and amputation depending on the site, and nephrectomy was performed in renal mucormycosis.

Fifty-year-old male with isolated renal mucormycosis admitted with complaints of fever, chills, pain over left lumbar region, and vomiting for 4 days. Ultrasonography abdomen showed evidence of pyelonephritis. Initially the patient was diagnosed to have pyelonephritis on ultrasound. Failure to respond to antibiotics with sterile blood and urine cultures led to further evaluation. The CT abdomen revealed left pyelonephritis with obstructive uropathy. Double-J (DJ) stenting was done. Patient condition did not improve. He deteriorated progressively and then developed acute renal dysfunction. Due to impending respiratory failure, he was put under mechanical ventilation and CT-guided renal biopsy was done; and the histopathology of kidney showed extensive invasion with broad aseptate ribbon-like hyphae consistent with mucormycosis (Fig. 1). Liposomal amphotericin B injection was administered. Since there was no clinical improvement, left nephrectomy was done. Patient died postoperatively on the second day.

Thirty-five-year-old male who met with a road traffic accident had right brachial plexus injury; fractures of right clavicle, scapula, humerus, and multiple ribs; right hemopneumothorax; and hemoperitoneum. After treatment for cerebral edema and dyselectrolytemia, open reduction and internal fixation of humerus

Table 2: Distribution of various mucormycetes with various clinical presentations

\begin{tabular}{llllc}
\hline Isolates & Rhino-orbital & Cutaneous & Isolated renal & Total \\
\hline Rhizopus & 13 & - & 1 & 14 \\
Mucor & 3 & - & - & 3 \\
$\begin{array}{l}\text { Apophysomyces } \\
\text { variabilis }\end{array}$ & - & 5 & - & 5 \\
Lichtheimia & - & 1 & - & \\
corymbifera & & & & 1 \\
Unidentified & - & 2 & - & 2 \\
Total & 16 & 8 & 1 & 25 \\
\hline
\end{tabular}

Table 3: Treatment and outcome of mucormycosis cases

\begin{tabular}{llllllc}
\hline Management & Outcome & ROC & Cutaneous & Pulmonary & Isolated renal & Total \\
\hline Amphotericin B alone & Survived & 4 & - & 1 & - & 5 \\
& Died & 5 & - & - & - & 5 \\
Surgery & Survived & 1 & 2 & - & - & 3 \\
& Died & - & - & - & - & - \\
Both & Survived & 8 & 5 & - & - & 13 \\
& Died & 2 & - & - & - & 3 \\
None & Lost to follow-up & 8 & 1 & - & 9 \\
\hline
\end{tabular}




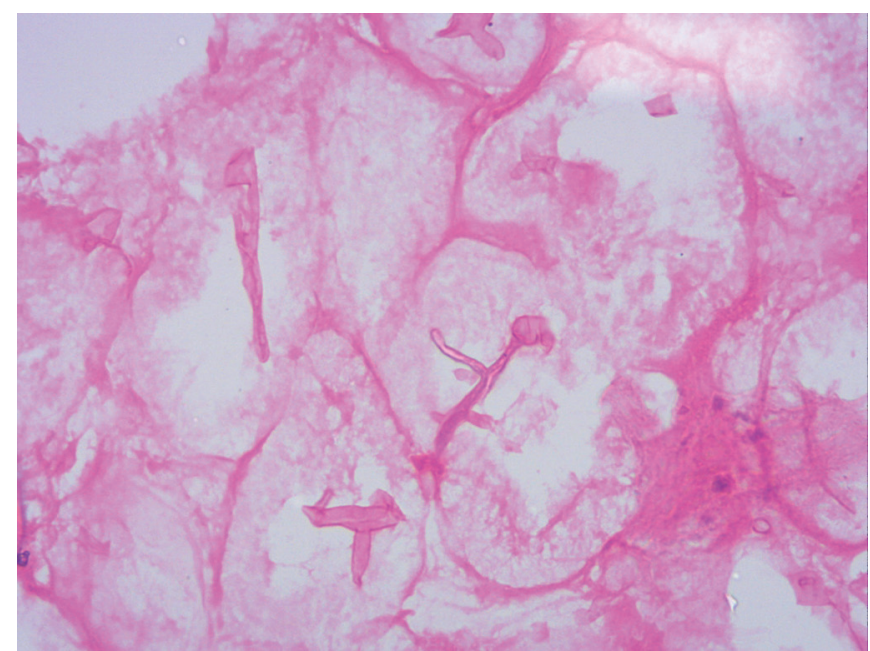

Fig. 1: Histopathology of kidney showing extensive invasion with broad aseptate ribbon-like hyphae consistent with mucormycosis

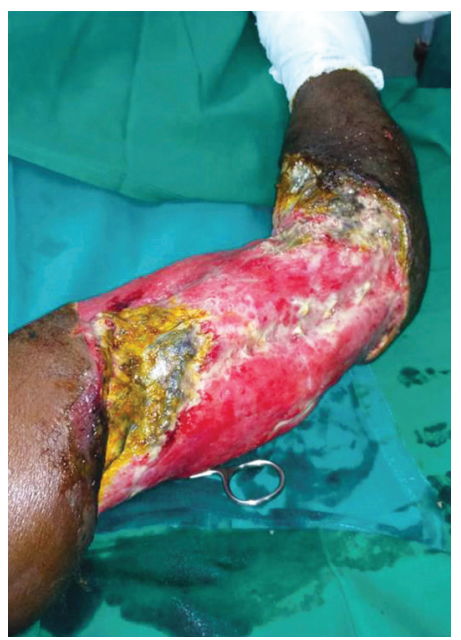

Fig. 3: Black necrosed tissue before starting antifungal therapy

and clavicle were done. On the 12th postoperative day, necrotizing fasciitis was observed and empirical amphotericin B was started. Debrided wound tissue showed bacterial growth and no evidence of fungi. Despite higher antibiotics, there was no clinical improvement. Deep tissue debridement was done and mycological techniques showed evidence of Apophysomyces elegans (Fig. 2). On 14th postoperative day, patient developed cardiac arrest, revived, and was put under mechanical ventilation. On 15th postoperative day, shoulder disarticulation was done and all necrotizing tissues were removed (Fig. 3). Conventional amphotericin B was administered twice daily for 30 days. Patient was discharged in good condition after 100 days of admission (Fig. 4).

\section{Discussion}

There has been a steady increase in reports of mucormycosis from different parts of the country. Variation in seasonal temperature and humidity in tropical areas may have direct impact on the growth of mucorales in the nature, and this may lead to seasonal fluctuation in the number of mucormycosis cases. In our study, increased number of cases was reported during the post rainy season.

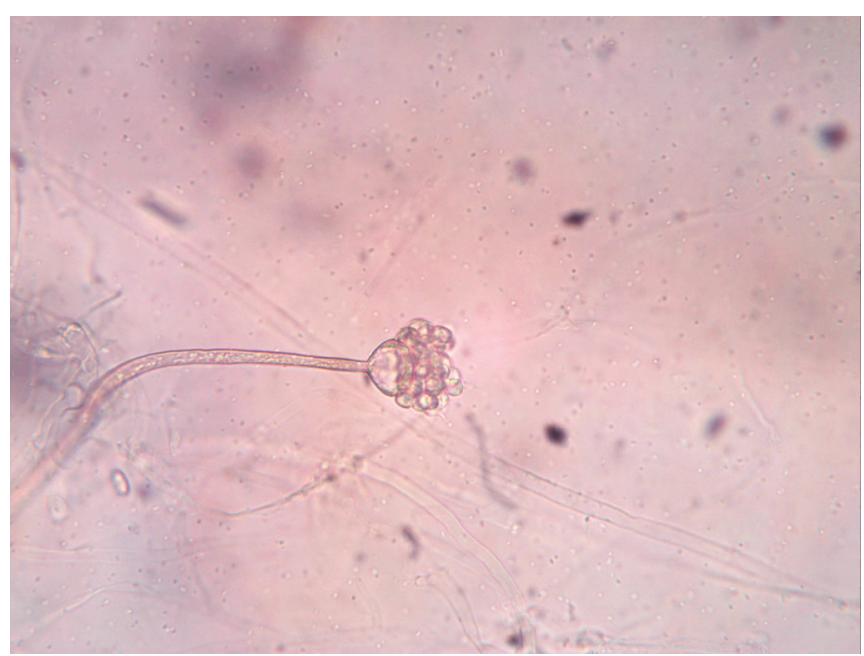

Fig. 2: Wet mount from water culture showing Apophysomyces variabilis

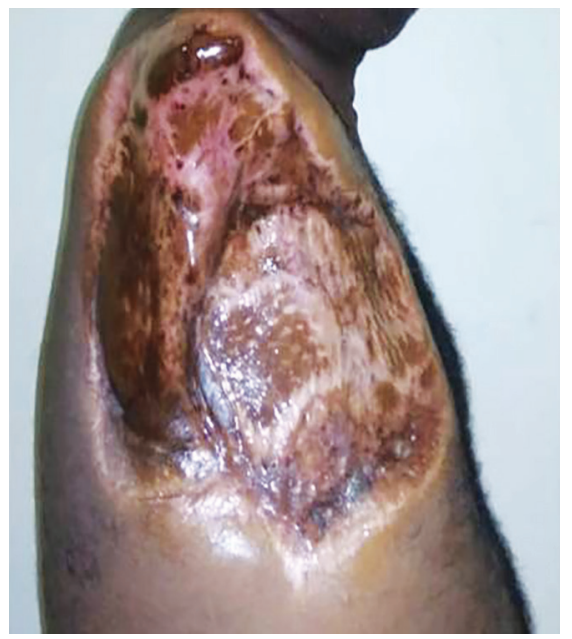

Fig. 4: After shoulder disarticulation and completion of 6 weeks of antifungal therapy

In India, mucormycosis cases are more prevalent in post rainy season and autumn months. ${ }^{1}$

As mentioned in previous Indian studies, ${ }^{2}$ uncontrolled diabetes mellitus (which seem to impair the ability of macrophages to prevent germination of sporangiospores) remains the most important risk factor for most of the cases. In India, considerable numbers (16 to $23 \%$ ) of diabetics remain undiagnosed before presentation of mucormycosis. ${ }^{3}$ Mucormycosis acted as diabetesdefining illness in those cases. In the present study, two such cases were identified. All cutaneous cases were immunocompetent with predisposing factors being road traffic accident and intramuscular injection in gluteal area. Risk factors associated with various clinical forms of mucormycosis are given in Table 4.

Worldwide, Rhizopus oryzae is the most common fungus isolated from clinical specimens of patients with mucormycosis followed by Lichtheimia corymbifera and Mucor racemosus. ${ }^{3}$ In the present study, most common fungus was Rhizopus oryzae followed by Apophysomyces variabilis, Mucor spp., and Lichtheimia corymbifera. Unlike other fungi which are globally distributed and reported predominantly in immunocompromised individuals, A. elegans has been reported mainly in warm climates mostly as 
Mucormycosis in South India

Table 4: Risk factors associated with various clinical forms of mucormycosis

\begin{tabular}{llllll}
\hline Risk factors & Rhino-orbital & Cutaneous & Pulmonary & Isolated renal & Total \\
\hline Diabetes mellitus & 26 & - & 1 & - & 27 \\
Trauma & - & 8 & - & - & 8 \\
Hematological malignancy & 2 & - & - & - & 2 \\
None & - & - & - & 1 & 1 \\
\hline
\end{tabular}

cutaneous infections after injury. Although it does not produce spores usually in the environment, there are case reports of systemic infection with this fungus. ${ }^{4}$

In disseminated mucormycosis, kidney is involved in $20 \%$ cases. $^{5}$ In contrary, isolated renal mucormycosis is increasingly being reported in India and China over the last two decades. ${ }^{6}$ In India, most patients were apparently normal individuals, whereas $70 \%$ of Chinese patients had underlying risk factors such as intravenous drug abuse, diabetes, kidney transplantation, and steroid therapy. Pathogenesis in renal mucormycosis is not clearly known. It is presumed that the infection could be from innocuous pulmonary lesion, as few patients had additional focus in lung on autopsy. ${ }^{7}$ In the present study, we had one case of isolated renal mucormycosis in a 50-year-old apparently immunocompetent male patient.

Early and accurate diagnosis is the most important factor for improving survival in patients with mucormycosis. In our study, the average duration of symptoms before presenting to hospital was 8 days in ROC cases and 14 days in cutaneous mucormycosis. Mean time taken for diagnosis of mucormycosis was 12 days in ROC cases and 28 days in cutaneous cases. In ROC cases, some clinical clues such as diplopia and necrotic naso-sinus eschars can be observed. Diagnostic delay was mainly observed in cutaneous infection presenting as necrotizing fasciitis, post-traumatic cellulitis, or injection abscess which are nonspecific manifestations and their differential diagnosis includes a range of infections caused by angioinvasive pathogens, including Aspergillus spp., Fusarium spp., Scedosporium spp., Pseudomonas aeruginosa, and nontuberculous mycobacteria. Therefore, a high index of suspicion is required for early recognition of mucormycosis. One important clue is progressive necrosis despite adequate medical therapy. Diagnosis of deep organ mucormycosis is even more challenging as in renal and pulmonary mucormycosis. ${ }^{8}$

All the cases were detected by direct wet mount, whereas the culture was positive only in $68 \%$ cases. Viability and recovery of the fungus from tissues can be attempted by careful mincing rather than using homogenizer. The infection is highly underreported due to nonspecific clinical symptoms and signs and difficulty in diagnosis. So autopsy is the gold standard approach for final diagnosis of mucormycosis, rate of which is declining globally.

Even in timely diagnosis, the window of opportunity is much shorter as they grow very rapidly in vivo. So effective treatment should be initiated before extensive angioinvasion occurs. ${ }^{9}$ Adding to the plight situation, they are resistant to many antifungals, including flucytosine, ketoconazole, fluconazole, voriconazole, and echinocandins with variable susceptibility to itraconazole. Amphotericin B, posaconazole, and ravuconazole are the only promising drugs. Combined medical and surgical intervention has improved survival rate over medical treatment alone. Very often extensive debridement of the sinonasal areas and orbit may be necessary which are cosmetically disfiguring and subsequent surgeries will be needed to rectify them. Due to prolonged antifungal therapy and surgical intervention, many patients from low socioeconomic status leave the facility despite medical advice. In the present study, of the 28 ROC cases, none of them underwent radical resection and 8 patients left against medical advice due to financial constraints.

\section{Conclusion}

To diagnose fungal infections promptly, a high index of suspicion in certain clinical settings cannot be overemphasized. Mucormycosis, an insidious killer, should be an important differential diagnosis in progressive soft tissue infections and deep organ infection. In India, it is likely that more cases can occur in the future due to increasing immunocompromised population, and it is the need of the hour that all medical fraternities of health community should have a firsthand knowledge of this organism to make a timely diagnosis and save patients' lives.

\section{References}

1. Nithyanandam S, Jacob MS, Battu RR, Thomas RK, Correa MA, D'Souza $\mathrm{O}$. Rhino-orbito-cerebral mucormycosis. A retrospective analysis of clinical features and treatment outcomes. Indian J Ophthalmol 2003;51(3):231-236.

2. Chakrabarti A, Das A, Mandal J, Shivaprakash MR, George VK, Tarai $B$, et al. The rising trend of invasive zygomycosis in patients with uncontrolled diabetes mellitus. Med Mycol 2006;44(4):335-342. DOI: 10.1080/13693780500464930.

3. Chakrabarti A, Chatterjee SS, Das A, Panda N, Shivaprakash MR, Kaur A, et al. Invasive zygomycosis in India: experience in a tertiary care hospital. Postgrad Med J 2009;85(1009):573-581. DOI: 10.1136/ pgmj.2008.076463.

4. Chakrabarti A, Shivaprakash MR, Curfs-Breuker I, Baghela A, Klaassen $H$, Meis JF. Apophysomyces elegans: epidemiology, amplified fragment length polymorphism typing, and in vitro antifungal susceptibility pattern. J Clin Microbiol 2010;48(12):4580-4585. DOI: 10.1128/JCM.01420-10.

5. Ingram CW, Sennesh J, Cooper JN, Perfect JR. Disseminated zygomycosis: report of four cases and review. Rev Infect Dis 1989;11(5):741-754. DOI: 10.1093/clinids/11.5.741.

6. Yu J, Li RY. Primary renal zygomycosis due to Rhizopus oryzae. Med Mycol 2006;44(5):461-466. DOI: 10.1080/13693780500338951.

7. Gupta KL, Khullar DK, Behera D, Radotra BD, Sakhuja V. Pulmonary mucormycosis presenting as fatal massive haemoptysis in a renal transplant recipient. Nephrol Dial Transplant 1998;13(12):3258-3260. DOI: $10.1093 / \mathrm{ndt} / 13.12 .3258$.

8. Georgiadou SP, Sipsas NV, Marom EM, Kontoyiannis DP. The diagnostic value of halo and reversed halo signs for invasive mold infections in compromised hosts. Clin Infect Dis 2011;52(9):1144-1155. DOI: 10.1093/ $\mathrm{cid} / \mathrm{cir} 122$.

9. Lewis RE, Lortholary O, Spellberg B, Roilides E, Kontoyiannis DP, Walsh TJ. How does antifungal pharmacology differ for mucormycosis versus aspergillosis? Clin Infect Dis 2012;54(Suppl 1):S67-S72. DOI: 10.1093/cid/cir884. 